tion of this lemma to the case where the coefficients of the system (5) and the initial data (6) depend on a parameter. The interval $(0,1)$ of course can be replaced by an arbitrary interval $(a, b)$, the initial values $\mathfrak{y}^{\prime}(0), \mathfrak{y}^{\prime \prime}(0)$ may be made distinct and the whole theory can be extended to systems of infinitely many equations, under suitable restrictions upon the matrices and vectors involved.

BROWN UNIVERSITY

\title{
ON THE NUMBER OF APPARENT MULTIPLE POINTS OF VARIETIES IN HYPERSPACE
}

\author{
BY B. C. WONG
}

By an apparent point of multiplicity $s$ on a variety in $r$-space we mean a line which passes through a given point in $r$-space and meets the variety in $s$ distinct points. In order that the number of such apparent $s$-fold points on a variety $V_{x}^{m}$ of order $m$ and of $x$ dimensions be finite, we must have $r=s t+1$, $x=(s-1) t$, where $t$ is the number less one of the hypersurfaces intersecting in the variety. In other words, the number of apparent $s$-fold points on a $V_{(s-1) t}^{m}$ which is the intersection of $t+1$ hypersurfaces in $S_{s t+1}$ is finite. It is the purpose of this paper to determine this number and also to determine its upper and lower limits.

We shall use the symbols $H_{s}^{(r)}, \bar{h}_{s}^{(r)}$ to denote respectively the maximum and minimum number of apparent $s$-fold points that a $V_{(s-1) t}^{m}$ of any order $m$ in $S_{r}[r=s t+1]$ can have, and the symbol $h_{s}^{(r)}$ to denote the number of those that a $V_{(s-1) t}^{m}$ of order $m=n_{1} n_{2} \cdots n_{t+1}$, which is the complete intersection of $t+1$ hypersurfaces of orders $n_{1}, n_{2}, \cdots, n_{t+1}$ respectively, ordinarily has. Thus if $s=2, t=1$ and therefore $r=3$, a curve $C^{m}$ in $S_{3}$ can have at most

$$
H_{2}{ }^{(3)}=(m-1)(m-2) / 2,
$$

and at least

$$
\bar{h}_{2}^{\left({ }^{(3)}\right.}=m(m-2) / 4, \quad \text { for } m \text { even, }
$$

and

$$
\bar{h}_{2}^{(3)}=(m-1)^{2} / 4, \quad \text { for } m \text { odd },
$$


apparent double points. If $C^{m}$ is the complete intersection of two surfaces of orders $n_{1}, n_{2}$ respectively, the number of apparent double points it has is

$$
h_{2}^{(3)}=n_{1} n_{2}\left(n_{1}-1\right)\left(n_{2}-1\right) / 2 .
$$

To illustrate further, let $s=r-1, t=1$. The number of apparent $(r-1)$-fold points on the complete intersection $V_{r-2}^{n_{1} n_{2}}$ of two hypersurfaces of orders $n_{1}, n_{2}$ respectively in $S_{r}$ is known to be*

$$
h_{r-1}^{(r)}=(r-1) !\left(\begin{array}{c}
n_{1} \\
r-1
\end{array}\right)\left(\begin{array}{c}
n_{2} \\
r-1
\end{array}\right)
$$

We shall show later that the maximum number of such points on a $V_{r-2}^{m}$ of any order $m$ is

$$
H_{r-1}^{(r)}=\left(\begin{array}{c}
m-r+2 \\
r-1
\end{array}\right)
$$

and we shall show that the minimum number on a $V_{r-2}^{m}$ of order $m=(r-1) m^{\prime}+k$, where $k$ may be any one of the integers from 0 to $r-2$, is

$$
\bar{h}_{r-1}^{(r)}=(r-1) !\left(\begin{array}{c}
m^{\prime} \\
r-1
\end{array}\right)+k(r-2) !\left(\begin{array}{c}
m^{\prime} \\
r-2
\end{array}\right) .
$$

We are to derive the formulas for $H_{s}^{(r)}, \bar{h}_{s}^{(r)}$, and $h_{s}^{(r)}$. For this purpose we make use of a method analogous to the one employed by the writer in the derivation of the formula for $h_{r-1}^{(r)}$ above in the paper referred to. We proceed to determine the formula for $h_{s}^{(r)}$ first.

Let the variety $V_{(s-1) t}^{m}\left[m=n_{1} n_{2} \cdots n_{t+1}\right]$ be the complete intersection of $t+1$ hypersurfaces of orders $n_{1}, n_{2}, \cdots, n_{t+1}$ respectively in $S_{r}[r=s t+1]$. Consider for a moment the hypersurfaces as being composed entirely of hyperplanes: $a_{1}^{(1)}$, $a_{2}^{(1)}, \cdots, a_{n_{1}}^{(1)} ; a_{1}^{(2)}, a_{2}^{(2)}, \cdots, a_{n_{2}}^{(2)} ; \cdots ; a_{1}^{(t+1)}, a_{2}^{(t+1)}, \cdots, a_{n_{t+1}}^{(t+1)}$. Then $V_{(s-1) t}^{m}$ is composed of $n_{1} n_{2} \cdots n_{t+1}(s-1) t$-spaces

$$
a_{i_{1}}^{(1)} a_{i_{2}}^{(2)} \cdots a_{i_{t+1}}^{(t+1)}, \quad\left[i_{j}=1,2, \cdots, n_{j}\right] .
$$

We write $\left(x_{1}, x_{2}, \cdots, x_{t+1}\right)$ in place of $a_{i_{1}}^{(1)} a_{i_{2}}^{(2)} \cdots a_{i_{t+1}}^{(t+1)} \cdots$ and

$*$ B. C. Wong, On the number of apparent triple points of surfaces in space of four dimensions, this Bulletin, vol. 35, pp. 339-343. 
regard $x_{1}, x_{2}, \cdots, x_{t+1}$ as the cartesian coordinates of a point in a $(t+1)$-space. Removing the restriction that the hypersurfaces be made up of hyperplanes, we take the totality of the $m=n_{1} n_{2} \cdots n_{t+1}$ points $\left(x_{1}, x_{2}, \cdots, x_{t+1}\right)$ for all the integral values of $x_{j}$ from 1 to $n_{j}[j=1,2, \cdots, t+1]$ to be the symbolic representation of $V_{(s-1)}^{m}$, proper or improper. In this representation any point or any set of values of $\left(x_{1}, x_{2}, \cdots, x_{t+1}\right)$ taken alone means an $S_{(s-1) t}$ forming a part of $V_{(s-1) t}^{m}$ and, in general, if we divide the total group of the $m$ points in any way into two or more groups, then these groups represent two or more varieties into which $V_{(s-1) t}^{m}$ degenerates. Any group of $s$ points with all of the $t+1$ corresponding coordinates chosen different, as $(1,1, \cdots, 1),(2,2, \cdots, 2), \cdots,(s, s, \cdots, s)$ or $(1,2$, $3, \cdots, t+1),(2,3,4, \cdots, 1),(3,4,5, \cdots, 2), \cdots,(s, s+1$, $s+2, \cdots, s-1)$ represents $s(s-1) t$-spaces which are not concurrent. Since only one line can be drawn passing through a given point and meeting each of $s(s-1) t$-spaces in general positions, the existence of such a group of $s$ points in the symbolic representation of $V_{(s-1) t}^{m}$ means the existence of an apparent $s$-fold point on $V_{(s-1) t}^{m}$. The total number of such groups is the number $h_{s}^{(r)}$ we are seeking.

To derive the formula for $h_{s}^{(t)}$ we first consider a group $G$ of $s^{t+1}$ points $\left(x_{1}, x_{2}, \cdots, x_{t+1}\right)$ in the representation of $V_{(s-1) t}^{m}$ whose coordinates take on all the possible sets of values formed from any $s$ positive integers not exceeding $n_{j}[j=1,2, \cdots, t+1]$ with repetitions allowed. Thus one such group $G$ for $s=2, t=2$ consists of the following 8 points $(1,1,1),(2,1,1),(1,2,1)$, $(1,1,2),(2,2,1),(2,1,2),(1,2,2),(2,2,2)$. Then the question is, How many groups of $s$ points all of whose corresponding coordinates are different are there contained in a group $G$ of $s^{t+1}$ ? The answer is obviously $N=(s !)^{t}$. Thus for $s=t=2$, the number of pairs of points with corresponding coordinates different is 4 and they are $(1,1,1),(2,2,2) ;(2,1,1),(1,2,2)$; $(2,2,1),(1,1,2) ;(2,1,2),(1,2,1)$.

Next we ask the question: How many groups of the type $G$, each consisting of $s^{t+1}$ points, as defined above, are contained in the total representation of $V_{(s-1) t}^{m}$ ? Since each $x_{j}$ may take on all the integral values from 1 to $n_{j}$, the total number $N^{\prime}$ of $G$ is $\left(\begin{array}{l}n_{1} \\ s\end{array}\right)\left(\begin{array}{l}n_{2} \\ s\end{array}\right) \cdots\left(\begin{array}{l}n_{t+1} \\ s\end{array}\right)$ as each $G$ involves all $s$ of the integral values. 
Hence the total number of groups of $s$ points with all corresponding coordinates unlike is the product $N N^{\prime}$, that is,

$$
h_{s}^{(r)}=(s !)^{t}\left(\begin{array}{c}
n_{1} \\
s
\end{array}\right)\left(\begin{array}{c}
n_{2} \\
s
\end{array}\right) \cdots\left(\begin{array}{c}
n_{t+1} \\
s
\end{array}\right)
$$

which is the required formula. If $s=2, t=1$, or if $s=r-1, t=1$, we have formulas (3) or (4) above, respectively.

Now to determine $H_{s}^{(r)}$, that is, the greatest number of apparent $s$-fold points a $V_{(s-1) t}^{m}$ can have. To give concreteness to the reasoning, we proceed to derive formula (5) for $H_{r-1}^{(r)}$. In this case $s=r-1, t=1$. The variety $V_{r-2}^{m}$ must be the partial intersection of two hypersurfaces and the $m$ points $\left(x_{1}, x_{2}\right)$ in the symbolic representation of the variety must be such that every two consecutive points have one of the coordinates take on values alike and the other take on different values. One such variety may be represented by the following $m$ points: $(1,1)$, $(1,2),(2,2),(2,3), \cdots,(m / 2,(m+2) / 2)$ if $m$ is even or $(1,1),(1,2),(2,2),(2,3), \cdots,([m+1] / 2,[m+1] / 2)$ if $m$ is odd. In either case, the total number of groups of $r-1$ points whose corresponding coordinates are all different is

$$
H_{r-1}^{(r)}=\left(\begin{array}{c}
m-r+2 \\
r-1
\end{array}\right)
$$

which is formula (5) above. If $r=3$, this is reduced to formula (1).

Tofind $H_{s}^{(r)}$ we reason in an analogous way. The variety $V_{(s-1) t}^{m}$ is the partial intersection of $t+1$ hypersurfaces. The representation of this variety consists of $m$ points such that every group of $q[q=2,3, \cdots, s+1]$ points has $q-1$ corresponding coordinates take on different values. For example, if $t=3, V_{3(s-1)}^{m}$ may be represented by the following $m$ points: $(1,1,1,1),(2,1,1,1)$, $(2,2,1,1),(2,2,2,1),(2,2,2,2),(3,2,2,2),(3,3,2,2)$, etc. It can be easily verified that the total number of groups of $s$ points with the corresponding coordinates all different is

$$
H_{s}^{(r)}=\left(\begin{array}{c}
m-s t+t \\
s
\end{array}\right) \text {. }
$$

This formula is reduced to (5) if $s=r-1, t=1$ and to (1) if $s=2, t=1$. 
To find $\bar{h}_{s}^{(r)}$, we notice that, if the number of apparent $s$-fold points on a $V_{(s-1) t}^{m}$ is to be minimum, the variety must be the complete or a partial intersection of $t+1$ hypersurfaces, $t$ of which are of order $s .{ }^{*}$ Putting $n_{1}=n_{2}=\cdots=n_{t}=s, n_{t+1}=m / s^{t}$ in formula (7) above, we have

$$
\bar{h}_{s}^{(r)}=(s !)^{t}\left(\begin{array}{c}
m / s^{t} \\
s
\end{array}\right)
$$

for $m$ a multiple of $s^{t}$. If $m$ is not a multiple of $s^{t}$, let $m=s^{t} m^{\prime}+k$ where $k$ may be zero or any of the integral values from 1 to $s^{t}-1$. Substituting in (7), we have

$$
(s !)^{t}\left(\begin{array}{c}
(m-k) / s^{t} \\
s
\end{array}\right) \text { or }(s !)^{t}\left(\begin{array}{c}
m^{\prime} \\
s
\end{array}\right)
$$

to which is to be added

$$
k[(s-1) !]^{t}\left(\begin{array}{c}
(m-k) / s^{t} \\
s-1
\end{array}\right) \quad \text { or } k[(s-1) !]^{t}\left(\begin{array}{c}
m^{\prime} \\
s-1
\end{array}\right),
$$

which is the number of groups of $s$ points with the $t+1$ corresponding coordinates different, each group containing one and only one of the $k$ points in the symbolic representation of $V_{(j-1) t}^{m}$. Hence

$$
\left\{\begin{aligned}
\bar{h}^{(r)} & =(s !)^{t}\left(\begin{array}{c}
(m-k) / s^{t} \\
s
\end{array}\right)+k[(s-1) !]^{t}\left(\begin{array}{c}
(m-k) / s^{t} \\
s-1
\end{array}\right) \\
& =(s !)^{t}\left(\begin{array}{c}
m^{\prime} \\
s
\end{array}\right)+k[(s-1) !]^{t}\left(\begin{array}{c}
m^{\prime} \\
s-1
\end{array}\right)
\end{aligned}\right.
$$

where $m^{\prime}=(m-k) / s^{t}$. If $k=0$, this is reduced to (9). If $s=2$, $t=1$, we have (2) and if $s=r-1, t=1$, we have (6).

The University of California

* If any of the hypersurfaces is of order less than $s$, then $\bar{h}_{s}^{(r)}=0$. 